\title{
Les effets d'une approche pédagogique préconisant la psychopédagogie du bienêtre sur la réussite éducative d'élèves du primaire
}

\author{
Auteurs \\ Nancy Goyette, Ph. D., professeure et chercheure au Département des \\ sciences de l'éducation, Université du Québec à Trois-Rivières, Canada, \\ nancy.goyette@uqtr.ca
}

Stéphane Martineau, Ph. D., professeur et chercheur au Département des sciences de l'éducation, Université du Québec à Trois-Rivières, Canada, stephane.martineau@uqtr.ca

Brigitte Gagnon, D. Ed., conseillère pédagogique au Centre de services scolaire des Hautes-Rivières, Canada,

brigitte.gagnon@csdhr.qc.ca

Julie Bazinet, M. Ed., conseillère pédagogique au Centre de services scolaire des Hautes-Rivières, Canada,

julie.bazinet@csdhr.qc.ca 


\section{REVUE HYBRIDE DE L'ÉDUCATION}

\section{Résumé}

Au Québec (Canada), la réussite éducative est une approche préconisée par une récente politique gouvernementale « qui va au-delà de l'obtention du diplôme en recherchant l'atteinte du plein potentiel de la personne dans toutes ses dimensions, sans égard à sa provenance, à son milieu ou à ses caractéristiques » (Gouvernement du Québec, 2016). Dans cette perspective, une recherche-action inspirée du modèle de Guay et Prud'homme (2011), menée avec 5 enseignantes d'une école primaire, a permis l'émergence d'une communauté d'apprentissage (Dionne, Lemyre et Savoie-Zajc, 2010) préconisant la psychopédagogie du bienêtre (Goyette, Gagnon, Bazinet et Martineau, 2020) Ce nouveau concept intègre des interventions pédagogiques bienveillantes en contexte scolaire, afin d'aider les élèves à ressentir du bienêtre, à s'épanouir et à fonctionner de manière optimale dans diverses situations en contexte scolaire. Un devis méthodologique mixte a permis de recueillir des données auprès des élèves ( $n=265$ ) par un questionnaire ainsi que par des entrevues individuelles et des entretiens collectifs actifs auprès des enseignantes. Cet article présentera les démarches entreprises par la communauté d'apprentissage afin d'identifier certains effets des interventions pédagogiques bienveillantes sur le bienêtre des élèves à l'école, sur leur capacité à nommer leurs forces et des moyens afin de ressentir du bienêtre et sur leur réussite éducative.

Mots-clés: psychopédagogie du bienêtre; recherche-action; réussite éducative; interventions pédagogiques; enseignement primaire 


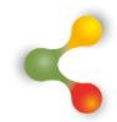

\section{REVUE HYBRIDE DE L'ÉDUCATION}

\section{Problématique}

Le grand chantier en éducation amorcé lors de la Révolution tranquille par le dépôt du rapport Parent (1963) a favorisé l'entrée du Québec dans la modernité et celle de la démocratisation de l'éducation. Étant un élément central qui a guidé les réflexions et les recommandations qui ont mené à plusieurs changements que l'on connait aujourd'hui, la démocratisation de la fréquentation scolaire avait comme objectif la généralisation d'une formation de base uniforme sur le plan des savoirs et des valeurs, pour que l'école devienne, d'une part, un élément de cohésion sociale et que d'autre part, elle puisse fournir à la société des ressources humaines compétentes pour assurer le développement des sous-systèmes politique, économique, culturel, démographique, social, etc. (Rocher, 2004) Depuis, plus que jamais, la scolarisation des individus s'avère nécessaire à leur développement dans une société où les emplois deviennent de plus en plus spécialisés et diversifiés pour répondre aux besoins des industries et de nouveaux secteurs d'activités reliés à l'information, aux communications, à l'informatique et à la technologie (Tardif et Lessard, 2004).

Toutefois, un enjeu considérable vient assombrir les grandes aspirations émanant de cette idéologie : ce ne sont pas tous élèves qui réussissent à terminer leurs études secondaires, bien que le taux de diplomation soit en constante évolution. Selon le rapport Diplomation et qualification par commissions scolaires au secondaire (gouvernement du Québec, 2019), le taux de diplomation total en 2017-2018 se chiffre à $81,6 \%$ (86,1\% pour les filles et $77,6 \%$ pour les garçons). Dans la foulée de ces statistiques, la réussite éducative est devenue une approche préconisée par une récente politique gouvernementale «qui va au-delà de l'obtention du diplôme en recherchant l'atteinte du plein potentiel de la personne dans toutes ses dimensions, sans égard à sa provenance, à son milieu ou à ses caractéristiques » (gouvernement du Québec, 2017, p. 1). Selon Lapostolle (2006, p. 7), contrairement à la réussite scolaire qui se mesure par « les résultats, les diplômes obtenus à la fin d'un cours ou d'un programme ", la réussite éducative est encore un concept relativement flou et peu documenté, s'appréhendant par des indicateurs d'ordre qualitatif. La réussite éducative vise, d'une part le développement harmonieux et dynamique de l'élève dans toutes ses potentialités que ce soit à l'école ou dans son milieu de vie (famille), mais aussi d'autre part, elle renvoie au développement de la citoyenneté (périscolaire) (Potvin, 2010). Loin d'en être dissociée, la réussite éducative est liée à la réussite scolaire laquelle constitue l'un de ses éléments (Bourgeois, 2010).

Pour atteindre un taux de diplomation de $90 \%$ avant 2030, la Politique de la réussite éducative (gouvernement du Québec, 2017) revisite le concept de démocratisation en se tournant vers des valeurs plus 


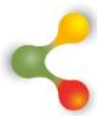

\section{REVUE HYBRIDE DE L'ÉDUCATION}

humanistes telles que l'universalité, l'accessibilité et l'équité (gouvernement du Québec, 2017), et en préconisant a) l'atteinte du plein potentiel de tous (axe 1) ; b) la mise en place d'un milieu inclusif propice au développement, à l'apprentissage et à la réussite (axe 2) et c) la mobilisation des acteurs et des partenaires pour la réussite (axe 3). Cette réorientation quant aux valeurs et aux axes privilégiés pour atteindre les objectifs ministériels tend vers une préoccupation grandissante envers le bienêtre à l'école, qui semble l'une des pistes de solution. Selon plusieurs recherches (Pinel-Jacquemin, 2016; Rascle et Bergugnat, 2016; Seligman, Ernst, Gillham, Reivich et Linkins, 2009), les élèves qui éprouvent du bienêtre à l'école apprennent mieux et persévèrent plus dans leur cheminement scolaire.

Partant de ce constat, dans de nombreux pays occidentaux (Australie, Angleterre, États-Unis), plusieurs programmes d'éducation positive ont été créés et intègrent divers concepts associés au bienêtre (ex. : compétences émotionnelles, forces de caractère, gratitude) et à son apprentissage (Shankland et Rosset, 2017). Ces programmes ont parfois été imposés ou proposés comme une démarche à appliquer de façon technique aux enseignants et aux intervenants. Or, l'imposition de programmes, de modèles ou d'injonction aux professionnels du monde scolaire ne serait pas la meilleure méthode pour développer une école qui promeut le développement du bienêtre (Rascle et Bergugnat, 2016). En effet, l'enjeu autour de la question du bienêtre à l'école requiert une réflexion collective et contextualisée des divers intervenants par rapport au sens, aux valeurs et aux actions à entreprendre. L'objectif est d'en venir, sinon à un consensus, à tout le moins à une entente la plus large possible, sur ce qui favorise véritablement un changement de perspective et un engagement des divers acteurs éducatifs envers le bienêtre. C'est dire qu'on ne saurait négliger le rôle des enseignants d'autant plus que ce sont eux qui interviennent directement et quotidiennement auprès des élèves et, par conséquent, qui sont les mieux à même d'intégrer des dispositifs pédagogiques orientés vers le bienêtre en classe (Goyette, 2018b). II est alors permis de s'interroger sur les types de moyens à mettre en œuvre pour permettre aux enseignants de développer un climat de classe favorable au bienêtre tout en préservant leur autonomie professionnelle.

Ce texte présente une recherche-action (2016-2019) s'échelonnant sur trois années qui a permis à des enseignantes du primaire faisant partie d'une communauté d'apprentissage de développer des activités pédagogiques favorisant le bienêtre de leurs élèves. Les résultats présentés dans ce texte permettront de répondre aux deux questions suivantes :

1) Qu'en est-il du sentiment de bienêtre de ces élèves à l'école qui évoluent dans un environnement favorisant la bienveillance? 


\section{8}

\section{REVUE HYBRIDE DE L'ÉDUCATION}

2) Quels sont les effets d'interventions pédagogiques ciblées en classe visant le bienêtre et la réussite éducative des élèves?

Or, cette problématique du bienêtre est au cœur d'un champ de recherche : la psychologie positive. Voyons-en certaines dimensions.

\section{Cadre conceptuel}

\section{La psychologie positive}

Globalement, le champ de la psychologie positive (Seligman et Csikszentmihalyi, 2000) étudie depuis une vingtaine d'années les traits de personnalité, les attitudes ainsi que les valeurs des individus pour les aider à se construire des forces et des compétences psychologiques pour un développement optimal de son bienêtre (Seligman, 2011). Gable et Haidt (2005) font écho à Seligman et Csikszentmihalyi (2000) en la définissant comme l'étude des processus et des conditions favorisant le fonctionnement optimal des individus, des groupes, et des organisations. À cet égard, le bienêtre est l'un des concepts centraux en psychologie positive, bien qu'il n'y ait pas de consensus en ce qui a trait à une définition universelle. II est l'objet de plusieurs recherches qui ont mené au développement de plusieurs théories, dont celle du bienêtre selon Seligman (2011). En s'appuyant sur le concept de prospérité, il définit le bienêtre par « l'atteinte d'un niveau optimal de fonctionnement d'un individu [...] qui se traduit par la bonté, la pérennité, la croissance personnelle et la résilience » (Goyette, 2014, p. 57).

\section{L'éducation positive}

Dans la foulée des recherches en psychologie positive, les chercheurs se sont intéressés au domaine de l'éducation, ce qui a donné lieu à l'éducation positive. Elle «tend à développer les compétences de bienêtre, d'épanouissement, et de fonctionnement optimal chez les enfants [...] au sein des institutions éducatives, réunissant ainsi fonctions préventives et fonctions habilitantes ou développementales " (Boniwell, 2011, p. 541). En contexte scolaire, Awartani, Vince Whitman et Gordon (2008) présentent trois types de bienêtre qui peuvent être ressentis par les élèves: le bienêtre physique, le bienêtre cognitif et le bienêtre psychosocial.

Le bienêtre physique fait référence au fait que les écoles doivent fournir un environnement sécuritaire aux élèves (bâtiments, terrains, trajet à effectuer pour y aller, l'équipement et le matériel mis à leur disposition). On parle aussi de politiques qui favorisent une bonne santé physique (activité physique quotidienne et bonne alimentation), ce qui aurait un lien avec de meilleurs apprentissages. 


\section{$\&$}

\section{REVUE HYBRIDE DE L'ÉDUCATION}

Le bienêtre cognitif, quant à lui, est en relation avec un contexte scolaire qui favorise le développement de stratégies d'apprentissage pour atteindre des objectifs pédagogiques, qui accompagne les élèves dans l'acquisition d'habiletés qui leur permettent de réaliser des tâches par l'expérimentation et la prise de risque. Un élève ressentant du bienêtre cognitif cultivera un sentiment de compétence et d'auto-efficacité positif qui lui permettra de réussir à l'école.

Le bienêtre psychosocial, quant à lui, regroupe le bienêtre social et émotionnel. Le bienêtre social permet entre autres aux élèves de développer des relations positives à l'école et de coopérer avec les autres. Le bienêtre émotionnel s'élabore par la prise en compte des émotions que l'on ressent et une saine gestion de ces dernières. La mise en œuvre d'un climat psychosocial positif à l'école favorisera ces deux éléments chez les élèves.

Pour cultiver un environnement bienveillant à l'école, un changement est souhaité en ce qui a trait aux pratiques enseignantes, pour passer d'un paradigme axé sur l'enseignement à celui axé sur l'apprentissage (Roegiers et Miled, 2012), paradigme qui prend en compte les disciplines scolaires ainsi que le développement de compétences émotionnelles, cognitives et psychosociales (Goyette, 2018a).

Les compétences émotionnelles « réfèrent aux différences dans la manière dont les individus identifient, expriment, utilisent et régulent leurs émotions et celles d'autrui » (Mikolajczak et Quoidbach, 2009, p. 7). Les compétences cognitives, quant à elles, interviennent dans le «processus mental lié à l'acquisition des connaissances, l'utilisation de l'information et le raisonnement» (Kiely, 2014) ${ }^{1}$. Elles sont rattachées aux fonctions cognitives telles que la mémoire, l'apprentissage et la prise de décision. Enfin, les compétences psychosociales aident à maintenir un état de bienêtre psychologique et à faire preuve d'une adaptation positive face aux différentes interactions avec son environnement social (Encinar, Tessier et Shankland, 2017).

\section{La psychopédagogie du bienêtre}

Dans le domaine des sciences de l'éducation, la psychopédagogie du bienêtre se définit comme un sous-champ de la psychopédagogie qui intègre la psychologie positive et la pédagogie, dont l'objet concerne le développement du bienêtre, l'épanouissement et le fonctionnement optimal chez les individus évoluant dans diverses situations pédagogiques. II vise le développement global de l'apprenant par l'intégration d'interventions pédagogiques bienveillantes, planifiées ou spontanées, favorisant l'appropriation de stratégies et d'habiletés propices à l'atteinte d'un équilibre émotif, cognitif, psychosocial et physique (Goyette, Gagnon,

\footnotetext{
${ }^{1}$ Traduction libre de l'anglais.
} 


\section{$\&$}

\section{REVUE HYBRIDE DE L'ÉDUCATION}

Bazinet et Martineau, 2020). Les trois années du projet ont permis le développement de la psychopédagogie du bienêtre, autant du côté des enseignantes (année 1), que du côté des élèves (année 2 et 3).

En conformité avec les questions de recherche identifiées plus haut, les objectifs de cette recherche étaient: 1) identifier le sentiment de bienêtre des élèves à l'école lorsqu'ils évoluent dans un environnement favorisant la bienveillance, et 2) identifier certains effets des interventions pédagogiques ciblées en classe visant le bienêtre et la réussite éducative des élèves.

\section{Quelques repères méthodologiques ${ }^{2}$}

Un devis séquentiel exploratoire (Fortin et Gagnon, 2016) a été mis en œuvre afin d'explorer le phénomène du bienêtre à l'école et les effets d'interventions pédagogiques ciblées en classe. Cette recherche-action a été menée avec cinq enseignantes, deux conseillères pédagogiques, une directrice d'une école et 265 élèves du primaire de la région de la Montérégie (Québec) et a permis l'émergence d'une communauté d'apprentissage préconisant la psychopédagogie du bienêtre (Goyette et al., 2020).

Pour identifier les effets du sentiment de bienêtre à l'école et des activités pédagogiques favorisant le bienêtre des élèves, les enseignantes ont eu à décider des moyens à entreprendre pour évaluer les effets des différentes interventions auprès de ceux-ci. Pour ce qui est du sentiment de bienêtre à l'école, elles ont opté pour la création d'un questionnaire qu'elles ont passé à la totalité des élèves de l'école. Pour ce qui est de l'évaluation des effets des activités pédagogiques sur le bienêtre des élèves et le possible lien avec la réussite éducative, les données sont issues d'entrevues et de questionnaires auprès des enseignantes.

\section{La recherche-action}

Ce type de recherche se définit comme «une pratique méthodologique centrée sur la résolution d'un problème concret vécu dans une situation pédagogique réelle dans le but d'y apporter des changements bénéfiques, de contribuer au développement professionnel des personnes qui y ont pris part et d'améliorer les connaissances sur cette situation " (Guay et Prud'homme, 2011, p. 188). Le choix d'une recherche-action est pertinent afin d'explorer les moyens mis en place par les enseignants pour intégrer le bienêtre dans leurs pratiques pédagogiques tout en développant leur identité professionnelle et leur autonomie. Le problème lié à la connaissance des concepts inhérents au bienêtre et à son intégration dans les pratiques pédagogiques a été un moteur riche en réflexion pour les

\footnotetext{
${ }^{2}$ Nous remercions monsieur Stéphane Thibodeau, professeur à l'Université du Québec à Trois-Rivières, pour sa collaboration à la rédaction de ce chapitre.
} 


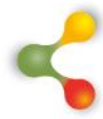

\section{REVUE HYBRIDE DE L'ÉDUCATION}

participantes pour les soutenir dans la réalisation d'actions concrètes, ce qui est l'une des caractéristiques essentielles de la recherche-action (idem, 2011). Une communauté d'apprentissage (Dionne, Lemyre et Savoie-Zajc, 2010) a été créée afin d'opérationnaliser cette recherche et de l'arrimer directement aux besoins spécifiques du milieu, tout en favorisant le développement professionnel des enseignantes.

\section{La communauté d'apprentissage}

Selon Dionne et al. (2010, p. 36), une communauté d'apprentissage est un dispositif qui comporte trois dimensions : une dimension cognitive, une dimension affective et une dimension idéologique. La dimension cognitive « vise le développement de la pratique pédagogique, l'acquisition d'un savoir individuel et collectif et la quête de sens ». Pour ce qui est de la dimension affective, elle se traduit par le fait que «la communauté d'apprentissage encourage l'enseignante au partage de savoirs et au soutien entre collègues ». Enfin, en ce qui a trait à la dimension idéologique, « la communauté d'apprentissage sert à l'émancipation des enseignants, par l'utilisation des recherches, en reconnaissant leur rôle dans la production de ces recherches, et elle vise ultimement à créer une cohésion et une vision commune dans l'école ". Dans ce projet, les différentes rencontres de la communauté visaient à favoriser et à soutenir la construction d'une vision commune de l'école en appuyant les interventions de tous les intervenants sur le concept de la bienveillance (dimension idéologique). Pour ce qui est des enseignantes qui participaient à la communauté, elles ont eu l'occasion de partager leurs idées et d'être soutenues par leurs collègues (dimension affective) afin de construire des activités signifiantes à intégrer dans leurs pratiques pédagogiques (dimension cognitive). De plus, soulignons que le travail de la communauté a permis de poser les premiers jalons de l'élaboration d'un nouveau champ en sciences de l'éducation : la psychopédagogie du bienêtre.

\section{Les objectifs annuels de la communauté}

La première année de la communauté a été consacrée au développement professionnel portant sur l'identité des enseignantes pour définir leur propre bienêtre en enseignement par des formations présentant certains concepts clés de la psychologie positive et explorer leurs motivations à intégrer le bienêtre quotidiennement en classe (Goyette, 2018b). Les travaux de la communauté ont aussi servi à définir ce que pouvait signifier enseigner le bienêtre aux élèves. Entre autres, cela consiste à supporter émotionnellement les élèves pour qu'ils développent des attitudes, des comportements et des compétences propices au bienêtre, à intégrer ce concept quotidiennement pour un meilleur transfert des apprentissages et, surtout, à ressentir soi-même du bienêtre au-delà de la complexité de la profession. À la fin de cette première année de projet, des entrevues individuelles ont permis l'exploration de leurs motivations à 


\section{8}

\section{REVUE HYBRIDE DE L'ÉDUCATION}

adopter des stratégies pédagogiques qui favorise le bienêtre des élèves (Goyette, 2018b).

Lors de la deuxième année de la communauté, les enseignantes ont développé des activités relatives aux concepts en psychologie positive qui suscitaient leur intérêt. Concrètement, des enseignantes se sont intéressées, entre autres, au développement des forces de caractère (Peterson et Seligman, 2004), à la tenue d'ateliers de philosophie (Lenoir, 2016), à la présence attentive (Malboeuf-Hurtubise et Lacourse, 2016), ainsi qu'à la mentalité de croissance (mindset) (Yeager et Dweck, 2012) dans le cadre d'activités avec leurs élèves. Pour ce faire, elles ont œuvré à les intégrer en contexte pédagogique lors de l'enseignement de disciplines tout en cultivant quotidiennement un sentiment de bienêtre à l'école pour leur bénéfice.

Enfin, lors de la troisième année, des activités pédagogiques élaborées l'année précédente ont été remises en place en plus d'en ajouter des nouvelles. Les enseignantes souhaitaient répertorier et formaliser la description des activités afin de les rendre disponibles pour leurs collègues de l'école. Un local propice au bienêtre comprenant différentes zones (lecture, yoga, écriture et dessin, pratique de la présence attentive, etc.) a également pris forme au sein de l'école pour vivre différentes activités sur ce thème.

\section{Les outils de collecte de données}

L'évaluation générale du bienêtre de ces élèves à l'école et des effets d'interventions pédagogiques ciblées en classe visant le bienêtre et la réussite éducative des élèves lors de cette recherche-action repose sur une diversité d'outils : des questionnaires électroniques, des entretiens individuels selon la méthode de Seidman (2006) ainsi que des entretiens collectifs actifs (Boutin, 1997). La triangulation des données issus de plusieurs sources permettra de répondre aux questions de recherche pour décrire le phénomène (Creswell, 2003).

\section{Les questionnaires électroniques}

Tout au long de la recherche-action, des questionnaires électroniques comportant des réponses fermées et ouvertes (voir tableau 2 ), ont permis de collecter des données auprès des élèves (265) et des enseignantes (5). 


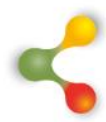

\section{REVUE HYBRIDE DE L'ÉDUCATION}

Tableau 1 : Répartition des élèves par niveau scolaire

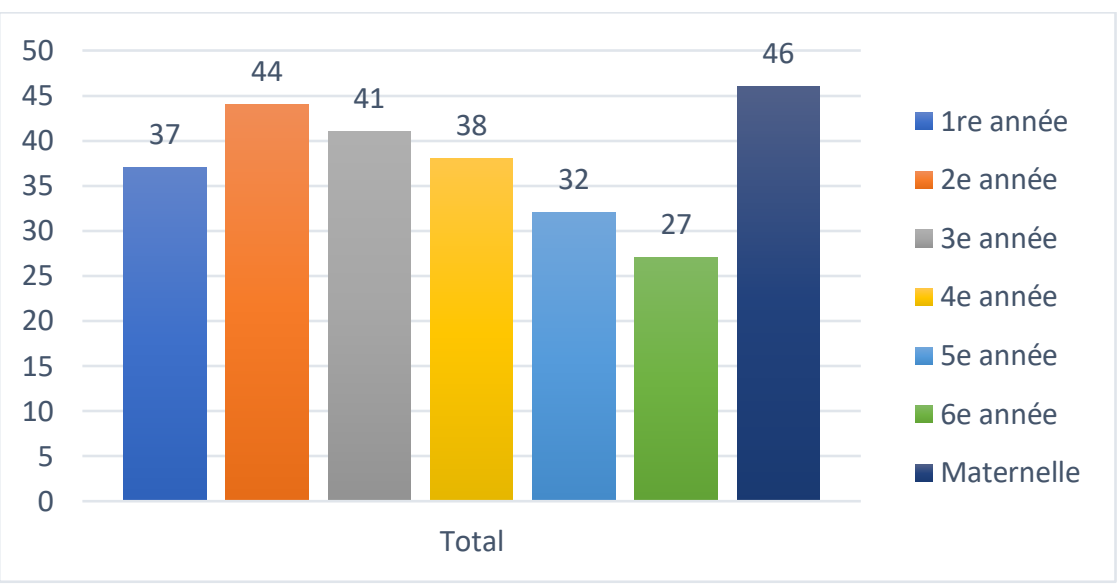

Un premier questionnaire a été utilisé auprès des élèves à la fin du projet pour répondre à la question 1 portant sur l'évaluation des effets des différentes interventions effectuées lors d'activités mobilisant tous les élèves de l'école (par exemple, la semaine de la gratitude). Ce questionnaire comportait 18 questions, dont 16 étaient fermées selon une échelle de likert de 4 (jamais [1], parfois [2], souvent [3], toujours [4]) et deux questions qui étaient ouvertes pour laisser les élèves inscrire leurs réponses personnelles. Les questions à choix de réponse ont été puisées à partir du questionnaire de Guimard, Ferrière, Bacro, Florin et Gaudonville (2012) sur le bienêtre à l'école et ont également été inspirées par les travaux de Encinar et al. (2017) pour mesurer certaines compétences psychosociales, cognitives et physiques. Ces trois indicateurs du bienêtre ont été mesurées à l'échelle de l'élève, de la classe et de l'école. Les deux questions ouvertes (questions 15 et 18) ont été créées en fonction du contexte précis des interventions entreprises auprès des élèves ; c'est-àdire en prenant en compte les activités spécifiques que les élèves ont expérimenté en classe. La passation des questionnaires électroniques auprès des élèves s'est effectuée entre le 13 et le 17 mai 2019 à l'aide d'un Ipad par le biais de l'application Google form.

Deux autres questionnaires ont été utilisés lors des années 1 et 2 du projet auprès des enseignantes pour constituer un bilan annuel. Les réponses ouvertes de ces derniers ont été l'objet d'une analyse thématique afin de répondre à la deuxième question de recherche.

\section{Les entretiens individuels et les entretiens collectifs}

Pour ce qui est de la deuxième question sur l'évaluation des effets des activités pédagogiques sur le bienêtre des élèves et le possible lien avec la réussite éducative, les données ont été issues d'entrevues semidirigées et de questionnaires auprès des enseignantes. Une série de 3 


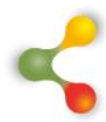

\section{REVUE HYBRIDE DE L'ÉDUCATION}

entrevues par enseignante selon la méthode de Seidman (2006) à différents moments de l'année scolaire (octobre/mars/mai) de la première année du projet a permis d'explorer leur parcours pour comprendre et établir leur profil d'enseignement prenant en compte le bienêtre de leurs élèves ${ }^{3}$. De plus, des entretiens (Boutin, 1997) ont aussi apporté des données supplémentaires à cette recherche. Par ce mode, il est possible de soutenir les participant.e.s (les cinq enseignantes) dans l'objectivation de leurs actions et dans leurs réflexions. Ce type d'entretien favorise la coconstruction des savoirs professionnels dans l'action.

Bien que des outils de collectes de données soient prévus dans la planification de la recherche-action, il importe de ne pas négliger les traces écologiques qui émergent en cours de projet. Par exemple, il peut être intéressant de prendre des photos des tableaux créés par les participants lors d'une réunion, de filmer une animation, de conserver un texte écrit par un participant, etc. Toutes ces traces pourront servir de preuves évocatrices lors de la diffusion du projet. Dans le cas de notre rechercheaction, différentes traces ont été recueillies : il s'agit de photos, de notes personnelles des membres de la communauté dont les cochercheurs, des canevas de travail, de travaux d'élèves, etc.

\section{Méthode d'analyse des données}

Les données quantitatives issues des questions fermées du questionnaire ont fait l'objet d'une analyse statistique descriptive selon la moyenne et une mesure de tendance centrale afin d'évaluer le sentiment de bienêtre général des élèves de cette école (Fortin et Gagnon, 2016). Le bienêtre a été analysé selon trois indicateurs (physiques, cognitifs et psychosociaux). Pour ce qui est des questions ouvertes du questionnaire (Q. 15 et 18) ainsi que des données issues des entrevues et des bilans écrits des enseignantes, elles ont été analysées selon l'analyse inductive générale (Blais et Martineau, 2006) par la création de catégories d'unité de sens répertoriées selon leur fréquence.

\section{Résultats}

\section{Analyse des questions fermées du questionnaire selon les 3 dimensions}

En faisant la moyenne des résultats pour chaque indicateur en considérant les réponses "souvent » et «toujours », lesquelles se situent à $85,24 \%$, les résultats issus des questionnaires démontrent que globalement, la majorité des élèves ressentent du bienêtre à l'école. Pour ce qui est des résultats concernant le bienêtre physique, $85,88 \%$ des élèves se sentent physiquement bien à l'école et éprouvent un sentiment

\footnotetext{
${ }^{3}$ Ces entrevues ont été faites dans le cadre d'une recherche en 2017-2018 financée par les Fonds d'innovation de la recherche (FIR) de l'UQTR.
} 


\section{REVUE HYBRIDE DE L'ÉDUCATION}

de sécurité. Puis, pour ce qui est du bienêtre cognitif, une moyenne de $84,71 \%$ des élèves apprécie globalement les activités à l'école et croit qu'ils peuvent y réussir. Enfin, la moyenne du bienêtre psychosocial des élèves se situe à $85,14 \%$, ce qui permet de constater que le milieu a mis en place des conditions favorables pour l'intégration des élèves dans le milieu et leur accompagnement. Rappelons ici que ces données ont été générées à la fin de la troisième année. Parce qu'il y a eu une seule prise de données, les résultats ne permettent pas de voir une évolution dans le temps. II s'agit à l'évidence d'une limite de la recherche.

Tableau 2 : Résultats du questionnaire selon les trois indicateurs du bienêtre

\section{Bienêtre physique} Jamais [1] Parfois [2] Souvent [3] Toujours [4]

1. Je me sens bien dans l'école. 2. Je me sens bien me rendant à l'école

3. Je me sens bien dans la classe

$\begin{array}{rrrr}1,64 \% & 8,42 \% & 31,93 \% & 58,01 \% \\ 3,77 \% & 8,30 \% & 21,89 \% & 66,04 \% \\ 4,15 \% & 9,43 \% & 21,89 \% & 64,53 \% \\ 4,15 \% & 14,72 \% & 28,68 \% & 52,45 \% \\ 2,64 \% & 7,92 \% & 20,75 \% & 68,68 \% \\ 3,04 \% & 11,15 \% & 33,11 \% & 52,70 \%\end{array}$

4. Je me sens bien aux récréations

5. Je me sens bien lors de mon retour à la maison

$3,04 \%$

$11,15 \%$

$33,11 \%$

$52,70 \%$

12. Les activités de détente m'aident à me sentir bien.

$3,97 \%$

$12,50 \%$

$32,09 \%$

$51,44 \%$

13. Je me sens bien quand je fais des activités physiques. $4,39 \% \quad 12,76 \%$

$34,52 \%$ $48,33 \%$

\section{Bienêtre cognitif}

6. J'aime les activités proposées dans l'école.

$\begin{array}{llll}3,21 \% & 11,57 \% & 33,56 \% & 51,66 \%\end{array}$

9. J'aime les activités proposées dans la classe.

$\begin{array}{llll}3,24 \% & 12,04 \% & 33,45 \% & 51,27 \%\end{array}$

14. Je sais que je peux réussir à l'école.

$3,66 \% \quad 11,64 \% \quad 33,51 \% \quad 51,19 \%$

15. Je connais mes forces et je peux en nommer.

$3,07 \%$

$12,73 \%$

$32,27 \%$ $51,93 \%$

Bienêtre psychosocial

7. J'ai des amis à l'école.

$3,64 \%$

$13,62 \%$

$33,89 \%$

$48,86 \%$

10. Je sens qu'on prend soin de moi à l'école. 


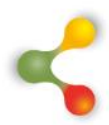

\section{REVUE HYBRIDE DE L'ÉDUCATION}

11. Je me sens encouragé(e) par les adultes lorsque que j'apprends.

16. Je suis à l'aise de parler de mes problèmes aux adultes.

17. Je suis fier(ère) de moi.

18. Je connais des trucs pour m'aider à me sentir bien.

\begin{tabular}{llll}
$2,69 \%$ & $11,32 \%$ & $34,30 \%$ & $51,68 \%$ \\
& & & \\
$2,94 \%$ & $10,28 \%$ & $33,78 \%$ & $53,00 \%$ \\
$2,87 \%$ & $12,14 \%$ & $33,22 \%$ & $51,77 \%$ \\
& & & \\
$3,83 \%$ & $11,87 \%$ & $31,64 \%$ & $52,66 \%$ \\
\hline
\end{tabular}

\section{Analyse des questions ouvertes}

En ce qui a trait à la question 15 , où les élèves devaient nommer leurs forces, presque la totalité a répondu à cette question $(n=262$, trois élèves n'ont pas répondu). Lors de l'analyse des données, nous avons retenu les réponses qui étaient associées à des forces liées à la personnalité des élèves (indicateurs cognitifs et psychosociaux) puisque cet aspect a été explicitement travaillé en classe. Les réponses reliées aux disciplines scolaires (ex. je suis bon.ne en français), au sport (ex. je suis bon.ne en baseball) ou aux passe-temps (ex. je suis bon.ne en dessin), ont été exclues de l'analyse, car elles portaient davantage sur le sentiment de compétence plutôt que sur le bienêtre. La force la plus saillante chez les élèves est la gentillesse. Elle inclut des sous-catégories telles qu' être aidant », «être serviable », «être généreux », «être encourageant » et "être ouvert". La deuxième force la plus grandement répertoriée est la persévérance. Puis, l'optimisme arrive en troisième et regroupe les souscatégories "souriant.e " et "être dynamique». L'établissement de relations positives est également une force soulignée par les élèves, qui se disent «sociables» et «à l'écoute des autres». Enfin, le courage est cinquième force nommée par ces derniers.

La question 18 a trait aux moyens que les élèves mettent en œuvre afin de se sentir bien. Les cinq moyens les plus utilisés par les élèves sont la respiration, parler à un adulte ou un ami, se retirer dans un endroit calme, dessiner/écrire/lire et utiliser des accessoires antistress (toutous, doudous, balles). On remarque plusieurs fois que les élèves utilisent une séquence d'action. Par exemple, respirer et lire ou se retirer, respirer et dessiner.

\section{Résultats des entrevues individuelles et des entretiens collectifs actifs chez les enseignantes}

Les résultats tirés d'une série de trois entrevues (Seidman, 2006) décrivent les motivations de ces enseignantes à préconiser des interventions pédagogiques (par exemple, ateliers philosophiques, ateliers sur les forces de caractère) favorisant l'intégration de la psychopédagogie du bienêtre en classe et leurs retombées chez les élèves selon elles (Goyette, 2018b). Les entretiens collectifs actifs ont enrichi leur propos 


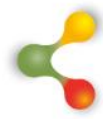

\section{REVUE HYBRIDE DE L'ÉDUCATION}

(Boutin, 1997). En effet, elles rapportent que les élèves ressentent du bienêtre dans leur classe et à travers les activités pédagogiques. L'aspect novateur et créatif associé à l'intégration des concepts de la psychologie positive dans leur classe est un élément qui alimente grandement leur motivation: "j'ai toujours une petite caractéristique que j'aime faire les choses autrement, là [...] je trouvais que c'était une belle opportunité de faire autrement [Entrevue 1-Monique] ${ }^{4} »$. D'ailleurs, elles rapportent le fait que cela leur permet de développer des relations positives avec les élèves, ce qui favorise le lien d'attachement qui est essentiel à l'apprentissage : "L'adaptation [scolaire] m'a permis de réaliser que, tant et aussi longtemps que le lien n'est pas créé avec les enfants, oublie les apprentissages [Entrevue 1-Monique]". Elles expriment aussi le besoin de les accompagner dans le développement de compétences personnelles et sociales qui les aideront à évoluer dans la vie puisque selon elles, ces interventions pédagogiques permettent aux élèves de développer leur estime de soi, ce qui contribue aux relations positives: "Quand tu as une bonne estime de toi-même, tu as confiance en toi, tu as confiance en la vie. [...] tu le sais que tu vas réussir à faire ton chemin, [...] tu vas y croire aussi. Puis tu vas développer [...] plus le temps va avancer, tu vas développer des bonnes relations. Tu vas créer autour de toi, la même chose que toi, ce que tu dégages [Entrevue 3-Nadia] ». Les interventions pédagogiques qu'elles mettent en place permettent aux élèves de ressentir des émotions positives, favorisent l'entraide et l'autonomie entre eux ainsi que la persévérance et l'optimisme accru.

Les moyens préconisés par ces dernières pour favoriser le bienêtre dans leur classe sont avant tout d'encourager les élèves à parler de leurs émotions: "Quand l'enfant est bien avec lui-même et y'apprend à se connaitre aussi, y'est plus ouvert à tout ce qui est autour de lui. [...] De lui offrir la possibilité de mettre des mots, de comprendre tout qu'il vit en dedans va peut-être l'aider à le régler plus facilement, aussi, et ensuite, à être plus apte à tout le reste autour, là, le plus académique [Entrevue1Marie] ». Elles soulignent l'importance de développer un climat de classe propice au bienêtre, ce qui favorise les apprentissages : " [...] être dans un bon climat de classe, comme ça, où on est calmes... souvent, tu vas rentrer dans ma classe, peu importe le moment, et c'est assez calme, généralement... [...] dans un milieu où on est calmes, ça favorise les apprentissages [Entrevue 1-Michelle]". La connaissance de leurs forces de caractère ${ }^{5}$ aide également les élèves à se construire une identité positive. Elles mettent également l'accent sur la nécessité de se recentrer sur soi lorsque certaines difficultés émotionnelles se présentent chez les élèves : "Le bienêtre des élèves qui sont anxieux fait que je repratique toujours la respiration, se calmer, prendre les choses une chose à la fois [Entrevue 3Sandrine] ». Enfin, partager sa gratitude quotidiennement est également un

\footnotetext{
${ }^{4}$ Les noms des participantes ont été changés pour garder l'anonymat.

${ }^{5}$ Selon Peterson et Seligman (2004), on répertorie 24 forces de caractère qui sont des traits cognitifs et non cognitifs positifs de la personnalité.
} 


\section{8}

\section{REVUE HYBRIDE DE L'ÉDUCATION}

moyen de favoriser le bienêtre chez les élèves. Les outils pédagogiques qu'elles ont utilisés pour en venir à ces moyens sont très variés. Toutefois, elles mettent en place, pour la majorité d'entre elles, la méditation et la pleine conscience, la rédaction d'un cahier de la gratitude, la cohérence cardiaque ainsi que l'utilisation de la littérature jeunesse sur des thèmes relatifs à l'éducation émotionnelle et à l'éducation positive.

Pour ce qui est de la réussite éducative, elles soulignent l'importance d'une démarche qui favorise le développement de compétences personnelles et sociales des élèves. Pour elle, la réussite académique n'est pas celle qu'il faut privilégier avant tout pour favoriser le bienêtre des élèves à l'école :

" Juste de préparer les élèves à avoir une attitude différente face à une évaluation, face à un travail qu'ils n'ont pas le goût de faire... Je trouve déjà ça amène du positif, fait que ça amène l'enfant à faire le travail d'une façon plus engagée. Fait que, souvent les résultats sont plus intéressants [Entrevue 1-Monique] ».

Selon elles, la réussite éducative est d'avant tout de développer des émotions positives en classe, d'inculquer la persévérance, ainsi que de développer des habiletés sociales pour bien évoluer en société. Leur mandat est donc de les guider dans le développement de leurs compétences émotionnelles :

"En tout cas, bref, oui la gestion des émotions. Quand tu sais que tu es capable, [...] quand tu vis des expériences qui t'amènent à régler tes choses, tu le développes ça. Mais ça, c'est pas inné chez les enfants [...]. [II] faut leur enseigner, faut leur montrer, faut l'appliquer. Quand tu prends conscience de comment tu es, comment les autres font, quand tu as conscience de l'autre, puis [que] tu as conscience de toi, tes actions vont aller en fonction de ce que tu es capable de comprendre [Entrevue 3- Nadia] ».

\section{Discussion}

Une analyse des résultats de cette recherche-action nous permet de répondre aux deux questions évoquées précédemment : 1) qu'en est-il du sentiment de bienêtre à l'école de ces élèves qui évoluent dans un environnement favorisant la bienveillance? 2) quels sont les effets d'interventions pédagogiques ciblées en classe visant le bienêtre et la réussite éducative des élèves? 


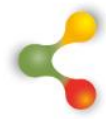

\section{REVUE HYBRIDE DE L'ÉDUCATION}

\section{Un environnement favorable à la bienveillance et le sentiment de bienêtre des élèves à l'école}

La bienveillance dans l'institution scolaire québécoise est un concept assez récent et polysémique (Bélanger et Royer, 2018). Actuellement, ce concept s'intègre graduellement dans les plans de réussite éducative de plusieurs commissions scolaires qui relient la notion de bienveillance au bienêtre des élèves. Elle est mise en lien avec des valeurs comme le respect, la coopération, la prise en compte de chacun et l'estime de soi et est une valeur qui guide les pratiques pédagogiques et professionnelles (Réto, 2018). Ces dernières requièrent une «posture, [des] attitudes et [des] dispositions essentiellement affectives à l'égard du receveur [c'est-àdire, l'élève] » (Bélanger et Royer, 2018, p. 5). Les résultats de cette recherche-action permettent de constater que l'environnement bienveillant dans lequel les élèves de cette école évoluent a eu un effet positif sur eux, que ce soit par rapport au bienêtre physique, cognitif ou psychosocial. Pour ce qui est du bienêtre physique, la moyenne des élèves se sent en sécurité dans leur milieu scolaire. Cela peut s'expliquer par les différentes activités offertes par l'école qui valorisent la bienveillance et la gratitude, entre autres la semaine de la gentillesse, qui a mobilisé tout le personnel et les élèves autour de ce thème. Cela explique en partie le fait que la force la plus identifiée par les élèves est la gentillesse. Elle inclut des souscatégories telles qu' "être aidant », "être serviable ", "être généreux », «être encourageant » et «être ouvert ».

D'autre part, le bienêtre cognitif est également élevé chez les élèves. L'un des facteurs est lié au fait que l'enseignement de la plupart des enseignants est teinté des concepts de la psychologie positive par l'intermédiaire de la communauté d'apprentissage. En effet, bien que certains élèves n'aient pas expérimenté les activités pédagogiques des enseignantes de la communauté durant ces trois années de façon formelle, ces dernières ont partagé à leurs collègues le fruit de leur travail (les activités) dans un fichier accessible à tous. Ce partage a permis aux élèves de construire un langage commun et de s'engager en cohérence avec les valeurs éducatives de l'école. Enfin, le bienêtre psychosocial a été développé à travers une culture qui valorise les relations positives, l'estime de soi et la régulation émotionnelle. Selon Bélanger et Royer (2018), «en contexte scolaire, il ne suffirait donc pas de simplement promouvoir la bienveillance comme une posture d'intervention, mais de concrètement soutenir une culture organisationnelle qui favorise l'émergence des sentiments qui y sont si intrinsèquement liés » (p. 5). La création du local Doudou est sans doute un bel exemple d'une initiative collective qui est en cohérence avec cette définition. 


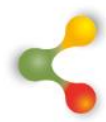

\section{REVUE HYBRIDE DE L'ÉDUCATION}

Les effets d'interventions pédagogiques pour favoriser la réussite éducative des élèves

La bienveillance étant au cœur du projet éducatif de cette école, les interventions pédagogiques des enseignantes de la communauté d'apprentissage ont été guidées par cette valeur. À cet égard, Bélanger et Royer (2018) rapportent les travaux de Noddings (1984) où elle "propose un modèle éducatif où, par apprentissage essentiellement vicariant » (Bandura, 1986), la bienveillance fait «boule de neige » au point où la réussite éducative est l'affaire de tous, et pour tous» (p. 5). La réussite éducative est perçue, par les enseignantes, comme le résultat d'un travail collectif qui permet le développement global de l'élève par la construction de compétences sociales et émotionnelles (Mikolajczak et Quoidbach, 2009) afin de les préparer à la vie en société. La réussite académique arrive au second plan puisqu'elle ne constitue pas nécessairement un élément essentiel pour elles. Ce qui importe, c'est que les élèves qui transitent par leur classe ressentent du bienêtre et évoluent en mettant à l'avant-scène la connaissance et l'estime de soi, ce qui leur permettra, par la suite de ressentir de la confiance en soi et du bienêtre. Or, les résultats de cette recherche-action mettent en exergue que la majorité des élèves sont capables de nommer une force qu'ils possèdent et détiennent des moyens pour les aider à gérer leurs émotions pour favoriser leur bienêtre. Le travail sur soi n'est pas inné chez les élèves et l'accompagnement fourni par les enseignantes leur a permis d'apprendre à ressentir du bienêtre pour favoriser leurs apprentissages. Pour ce faire, une relation de qualité doit être établie entre l'enseignante et les élèves (Rascle et Bergugnat, 2016). L'établissement de relations positives est également une force soulignée par les élèves, qui se disent " sociables » et «à l'écoute des autres ». Créer un lien significatif réciproque aide donc à développer des habiletés personnelles et sociales qui permettent la communication et la gestion des émotions. Ces deux éléments liés au développement de compétences émotionnelles (Mikolajczak et Quoidbach, 2009) sont centraux dans l'élaboration des interventions pédagogiques des enseignantes et les résultats recueillis auprès des élèves démontrent très bien qu'ils ont travaillé ces deux éléments puisqu'ils rapportent que parler à un adulte ou un ami (communiquer ses émotions) et se retirer dans un endroit calme (gérer ses émotions) constituent des moyens de les aider à ressentir du bienêtre.

On constate qu'instinctivement, les enseignantes mettent en application le concept de Caring (Noddings, 2002 dans Bélanger et Royer, 2018), qui se définit comme "étant avant tout une position d'écoute face aux besoins manifestés par l'élève ", et qui se transpose, selon ces auteurs, à la bienveillance. Elles ont mis en place ses quatre composantes : modeler, dialoguer, pratiquer et valider (idem, 2018). Par la composante "modeler ", elles incarnent les valeurs liées à la bienveillance et au bienêtre, par exemple, le respect, la persévérance et la gentillesse, auprès de leurs élèves. À cet égard, on remarque que la persévérance ressort 


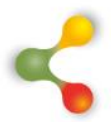

\section{REVUE HYBRIDE DE L'ÉDUCATION}

comme force dans leurs réponses. Puis, la composante « dialoguer » se constate par l'importance qu'accordent les enseignantes à l'établissement de liens significatifs par la communication constante avec les élèves pour mieux les connaitre et une posture de compréhension envers eux. La composante "pratiquer » réfère à l'intégration d'attitudes positives quotidiennement en classe, par de petits gestes. Les élèves rapportent qu'ils possèdent l'optimisme comme force, attitude que les enseignantes déclarent travailler avec leurs élèves. Elles ont conscience que le bienêtre et les attitudes positives ne s'apprennent pas formellement par des cours spécifiques. Enfin, la composante «valider» se retrouve fréquemment dans les propos des enseignantes, qui affirment encourager les élèves à se dépasser et à faire ressortir le meilleur d'eux-mêmes dans diverses situations qui génèrent parfois de l'anxiété. Elles proposent, pour ce faire, des moyens divers que les élèves peuvent utiliser librement au besoin. La question 18 apporte des données en ce qui a trait aux moyens que les élèves mettent en œuvre pour diminuer les émotions négatives: la respiration (méditation/pleine conscience), dessiner/écrire/lire, utiliser des accessoires antistress (toutous, doudous, balles), etc. On remarque toutefois que plusieurs élèves utilisent une séquence d'action. Par exemple, respirer et lire ou se retirer, respirer et dessiner. Cela peut s'expliquer par le fait qu'ils sont encouragés en classe à pratiquer ces moyens par les enseignantes et qu'ils en retirent des bénéfices, que ce soit à l'école ou à la maison.

\section{Limites de la recherche-action}

Bien que les résultats nous permettent de conclure que les interventions en psychopédagogie du bienêtre ont eu des effets positifs auprès d'élèves d'une école primaire, les données recueillies dans les questionnaires n'ont pas permis de discriminer les élèves des groupes des enseignantes prenant part à la communauté d'apprentissage. Elles ont donc servi à dresser un portrait global du bienêtre ressenti par tous les élèves de l'école. Comme il n'y a pas eu de collecte de données avant et après, il semble difficile de généraliser les résultats. Cependant, le questionnaire a permis à l'équipe-école de faire un portrait de situation actuelle auprès de l'ensemble de leurs élèves afin de voir sur quels aspects du bienêtre devraient porter les actions futures. On peut penser qu'un outil, tel que développé dans ce projet, pourrait être repris annuellement afin de réguler les actions en psychopédagogie du bienêtre auprès de l'ensemble des élèves ou dans certains groupes en particulier. De plus, ajoutons qu'en ce qui concerne l'évaluation des effets des activités pédagogiques déployées par les enseignantes de la communauté dans leur classe pour favoriser le bienêtre et la réussite éducative, les résultats s'appuient sur leurs perceptions, lesquelles (comme toute perception) peuvent être biaisées par le contexte et les préférences personnelles. Toutefois, ces résultats peuvent servir de tremplin pour une recherche qui irait au-delà des perceptions pour en arriver à une évaluation plus objective, par la construction d'outils permettant de mesurer les effets réels, nous pensons 


\section{$\&$}

\section{REVUE HYBRIDE DE L'ÉDUCATION}

ici notamment à des observations de pratiques ou à l'usage d'un questionnaire.

\section{Conclusion}

Notre texte a présenté une recherche-action que nous avons menée sur trois années. Nous avons tenté de répondre à deux principales questions. Rappelons-les ici : Qu'en est-il du sentiment de bienêtre des élèves à l'école qui évoluent dans un environnement favorisant la bienveillance? Quels sont les effets d'interventions pédagogiques ciblées en classe visant le bienêtre et la réussite éducative des élèves?

En ce qui concerne notre première interrogation, il ressort de notre recherche-action que le développement d'un environnement scolaire favorisant le sentiment de bienêtre chez les élèves nécessite : un plan d'action cohérent et explicite ainsi que la mobilisation de l'ensemble des intervenants. Les effets positifs enregistrés tiennent à l'évidence au fait qu'il ne s'agissait pas d'initiatives isolées, mais bien d'un projet auquel tout le personnel de l'école - et au premier chef les enseignantes - adhérait.

En ce qui a trait à notre deuxième interrogation, nos résultats tendent à démontrer que la mise en place de la méditation et la pleine conscience, la rédaction d'un cahier de la gratitude, la cohérence cardiaque ou encore l'utilisation de la littérature jeunesse sur des thèmes relatifs à l'éducation émotionnelle et à l'éducation positive constituent des outils pédagogiques pouvant favoriser la réussite éducative d'élèves du primaire (laquelle ne doit pas être confondue avec la réussite académique). Cette réussite éducative, ici, renvoie au fait de favoriser le développement des émotions positives, d'inculquer la persévérance et de permettre le développement des habiletés sociales. Comme nous l'avons vu, dans l'esprit des enseignantes rencontrées, réussite éducative et compétences émotionnelles sont étroitement liées.

En somme, notre recherche, modeste et exploratoire, tend à démontrer que la problématique du bienêtre est une question complexe et encore peu explorée. La psychopédagogie du bienêtre a justement pour finalité de mettre au jour des connaissances qui, non seulement permettront de comprendre quels sont les facteurs associés au bienêtre, mais aussi, qui favoriseront l'élaboration d'outils pédagogiques que les enseignants pourront s'approprier afin de soutenir le développement psychosocial et émotionnel des apprenants. 


\section{REVUE HYBRIDE DE L'ÉDUCATION}

\section{Références}

Bélanger, J. et Royer, F. (2018). Une bienveillance pour les élèves à besoin particulier au sein de l'école québécoise : un concept à nuancer. Questions Vives, 29. http://journals.openedition.org/questionsvives/3493

Awartani, M., Vince Whitman, C. et Gordon, J. (2008). Developing Instruments to Capture Young People's Perceptions of how School as a Learning Environment Affects their Well-Being. European Journal of Education Studies, 43(1).

Bandura, A. (1986). Social foundations of thought and action: A social cognitive theory. Prentice-Hall.

Blais, M. et Martineau, S. (2006). L'analyse inductive générale: description d'une démarche visant à donner un sens à des données brutes. Recherches qualitatives, 26(2), 1-18.

Boniwell, I. (2011). L'éducation positive: apporter le bien-être aux enfants et aux jeunes. Dans C. Martin-Krumm et C. Tarquinio (dir.), Traité de psychologie positive (p. 539-556). De Boeck.

Bourgeois, F. (2010). Définir la Réussite éducative ? Cahiers de l'action, 27(1), 57-72.

Boutin, G. (1997). L'entretien de recherche qualitatif. Presses de l'Université du Québec.

Commission royale d'enquête sur l'enseignement dans la province de Québec et Parent, A.- M. (1963). Rapport de la Commission royale d'enquête sur l'enseignement dans la province de Québec. Gouvernement du Québec. http://classiques.uqac.ca/contemporains/quebec_commission_par ent/commission_parent.html

Creswell, J. W. (2003). Research design : qualitative, quantitative, and mixed method approaches ( $2^{\mathrm{e}}$ éd.). Sage Publications.

Dionne, L., Lemyre, F. et Savoie-Zajc, L. (2010). Vers une définition englobante de la communauté d'apprentissage comme dispositif de développement professionnel. Revue des sciences de l'éducation, 36(1), 25-43.

Encinar, P.-E., Tessier, D. et Shankland, R. (2017). Compétences psychosociales et bien-être scolaire chez l'enfant: une validation française pilote. Enfance, 2017 (1), 37-60. 


\section{$\&$}

\section{REVUE HYBRIDE DE L'ÉDUCATION}

Fortin, M.-F. et Gagnon, J. (2016). Fondements et étapes du processus de recherche : méthodes quantitatives et qualitatives ( $3^{\mathrm{e}}$ éd.). Chenelière éducation.

Gable, S. L. et Haidt, J. (2005). What (and Why) Is Positive Psychology? . Review of General Psychology, 9, 103-110.

Goyette, N. (2014). Le bien-être dans l'enseignement : étude des forces de caractère chez des enseignants persévérants du primaire et du secondaire dans une approche axée sur la psychologie positive [thèse de doctorat inédite] Université du Québec à Trois-Rivières.

Goyette, N. (2018a). Enseigner le bien-être à l'école: une voie prometteuse et novatrice pour améliorer la réussite scolaire. Communication présentée Colloque innovation 2018, Lausanne, Suisse.

Goyette, N. (2018b). Les motivations d'enseignantes à adopter des interventions pédagogiques orientées vers le développement des compétences en littératie émotionnelle pour favoriser le bienêtre. Language et literacy, 20(1), 107-123.

Goyette, N., Gagnon, B., Bazinet, J. et Martineau, S. (2020). La communauté d'apprentissage au service du développement de l'agir compétent d'enseignantes en psychopédagogie du bien-être. Dans N. Goyette et M. Stéphane (dir.), Le bien-être en enseignement: tensions entre espoir et déceptions (p. 115-141.). Presses de l'Université du Québec.

Guay, M.-H. et Prud'homme, L. (2011). La recherche-action. Dans T. Karsenti et L. Savoie-Zajc (dir.), La recherche en éducation : étapes et approches ( $3^{e}$ éd.) (p. 183-211). ERPI.

Guimard, P., Ferrière, S., Bacro, F., Florin, A. et Gaudonville, T. (2012). Outils d'évaluation du bien-être à l'école. Centre de Recherche en Education de Nantes.

Kiely, K. M. (2014). Cognitive Function. Dans A. C. Michalos (dir.), Encyclopedia of Quality of Life and Well-Being Research. Springer.

Lapostolle, L. (2006). Réussite scolaire et réussite éducative: quelques repères. Pédagogie collégiale, 19(4), 5-7.

Lenoir, F. (2016). Philosopher et méditer avec les enfants. Albin Michel.

Malboeuf-Hurtubise, C. et Lacourse, É. (2016). Mission méditation : pour des élèves épanouis, calmes et concentrés. Midi trente éditions. 


\section{REVUE HYBRIDE DE L'ÉDUCATION}

Mikolajczak, M. et Quoidbach, J. (2009). Les compétences émotionnelles. Dunod.

Ministère de l'éducation et de l'enseignement supérieur. (2016). Politique de réussite éducative. Le plaisir d'apprendre, la chance de réussir. Gouvernement du Québec. http://www.education.gouv.qc.ca/fileadmin/site_web/documents/P SG/politiques_orientations/politique_reussite_educative_10juillet_ F_1.pdf

Ministère de l'éducation et de l'enseignement supérieur. (2019). Processus d'agrément des programmes de formation à l'enseignement. Gouvernement du Québec.

http://www.education.gouv.qc.ca/organismes-relevant-duministre/capfe/processus-dagrement-des-programmes-deformation-a-lenseignement/

Noddings, N. (1984). Caring. University of California Press.

Noddings, N. (2002). Starting at Home: Caring and social policy. University of California Press.

Peterson, C. et Seligman, M. E. P. (2004). Character Strengths and Virtues: a Handbook and Classification. Oxford University Press.

Pinel-Jacquemin, S. (2016). Le bien-être à l'école des enfants en situation de précarité. Conseil national d'évaluation du système soclaire. http://www.cnesco.fr/wpcontent/uploads/2017/10/170929 precarite.pdf

Potvin, P. (2010). La réussite éducative. www.pierrepotvin.com/6.\%20Publications/Textereussite\%20educative.doc

Rascle, N. et Bergugnat, L. (2016). Qualité de vie des enseignants en relation avec celle des élèves: revue de question, recommandations.

Réto, G. (2018). La bienveillance dans le champ scolaire. Caractérisation des pratiques et actualisation selon des membres du personnel enseignant de collège, des chefs d'établissement et des experts du monde de l'éducation. (Thèse inédite, Université catholique de l'Ouest (France) et Université de Sherbrooke (Canada)).

Rocher, G. (2004). Un bilan du Rapport Parent: vers la démocratisation. Bulletin d'Histoire politique, 12(2), 117-128. 


\section{REVUE HYBRIDE DE L'ÉDUCATION}

Roegiers, X. et Miled, M. (2012). Quelles réformes pédagogiques pour l'enseignement supérieur? : l'intégration des acquis, une piste pour placer l'efficacité au service de l'hunamisme. ( $1^{\mathrm{e}}$ éd.). De Boeck.

Seidman, I. E. (2006). Interviewing as Qualitative Research. A Guide for Researchers in Education and the Social Sciences. Teachers College Press.

Seligman, M. et Csikszentmihalyi, M. (2000). Positive Psychology. An Introduction. American Psychologist, 55(1), 5-15.

Seligman, M., Ernst, R., Gillham, J., Reivich, K. et Linkins, M. (2009). Positive education: positive psychology and classroom interventions. Oxford Review of Education, 35(3), 293-311. doi: $10.1080 / 03054980902934563$

Seligman, M. E. P. (2011). Flourish: A Visionary New Understanding of Happiness and Well-being. Free Press.

Shankland, R. et Rosset, E. (2017). Review of Brief School-Based Positive Psychological Interventions: a Taster for Teachers and Educators. Educational Psychology Review, 29(2), 363-392. doi: 10.1007/s10648-016-9357-3

Tardif, M. et Lessard, C. (2004). La profession d'enseignant aujourd'hui : évolutions, perspectives et enjeux internationaux. Les Presses de l'Université Laval.

Yeager, D. S. et Dweck, C. S. (2012). Mindsets that promote resilience: When students believe that personal characteristics can be developed. Educational Psychologist, 47(4), 302-314. 